\title{
Argininosuccinate lyase interacts with cyclin A2 in cytoplasm and modulates growth of liver tumor cells
}

\author{
YU-HSUAN HUNG ${ }^{1}$, HAU-LUN HUANG ${ }^{4}$, WEI-CHING CHEN ${ }^{1}$, MENG-CHI YEN ${ }^{5}$, CHIEN-YU CHO $^{1}$,

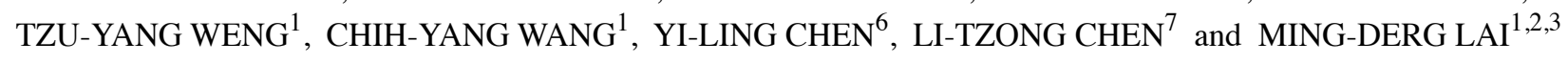 \\ ${ }^{1}$ Institute of Basic Medical Sciences, College of Medicine, National Cheng Kung University; \\ ${ }^{2}$ Department of Biochemistry and Molecular Biology, College of Medicine, National Cheng Kung University; \\ ${ }^{3}$ Center for Infectious Diseases and Signaling Research, College of Medicine, National Cheng Kung University, \\ Tainan 701; ${ }^{4}$ National Institute of Cancer Research, National Health Research Institutes, Miaoli 350; \\ ${ }^{5}$ Department of Emergency Medicine, Kaohsiung Medical University Hospital, Kaohsiung Medical University, \\ Kaohsiung 807; ${ }^{6}$ Department of Senior Citizen Services Management, Chia Nan University of Pharmacy and Science, \\ Tainan 717; ${ }^{7}$ National Institute of Cancer Research, National Health Research Institutes, Tainan 701, Taiwan, R.O.C.
}

Received July 31, 2016; Accepted December 13, 2016

DOI: $10.3892 /$ or.2016.5334

\begin{abstract}
Arginine is a critical amino acid in specific cancer types including hepatocellular carcinoma (HCC) and melanoma. Novel molecular mechanisms and therapeutic targets in arginine metabolism-mediated cancer formation await further identification. Our laboratory has previously demonstrated that arginine metabolic enzyme argininosuccinate lyase (ASL) promoted HCC formation in part via maintenance of cyclin A2 protein expression and arginine production for channeling to nitric oxide synthase. In this study, we investigated the mechanism by which ASL regulates cyclin A2 expression. We found that ASL interacted with cyclin A2 in HCC cells and the localization of their interaction was in the cytoplasm. Mutation of essential residues for enzymatic activity of ASL did not affect the binding of ASL to cyclin A2. Moreover, the mutant ASL retained the ability to restore the decreased tumorigenicity caused by ASL shRNA. Furthermore, overexpression of ASL conferred resistance to arginine deprivation therapy. Finally, the important pathways and potential therapeutic targets in ASL-regulated HCC were identified by bioinformatics analyses with Metacore database and Connectivity Map database. Our
\end{abstract}

Correspondence to: Professor Ming-Derg Lai, Department of Biochemistry and Molecular Biology, College of Medicine, National Cheng Kung University, 1 University Road, Tainan City 701, Taiwan, R.O.C.

E-mail: a1211207@mail.ncku.edu.tw

Abbreviations: ASL, argininosuccinate lyase; CCNA2, cyclin A2; 5-FU, 5-fluorouracil; ADI-PEG, arginine deiminase formulated with polyethylene glycol

Key words: argininosuccinate lyase, liver cancer, non-enzymatic function, cyclin A2, drug resistance, arginine deiminase analyses suggested that bisoprolol, celecoxib, and ipratropium bromide, are potential therapeutics for ASL-regulated HCC formation. Thus, ASL interacts with cyclin A2 in cytoplasm, and may promote HCC formation through this non-enzymatic function. Overexpression of ASL may be a contributing factor in drug resistance for arginine deprivation therapy.

\section{Introduction}

HCC is the fifth leading cause of cancer-related deaths in the world $(1,2)$. The high recurrence rate and poor prognosis of HCC are responsible for the high mortality resulted from this cancer. Surgery, loco-regional therapy, transcatheter arterial chemoembolization (3), and chemotherapy are available for HCC treatment, however, they provide limited success in reducing cancer-related mortality. Targeted therapy with multiple tyrosine kinase inhibitor sorafenib has improved HCC treatment (4), but it is of major concern to identify critical targets and underlying mechanisms involved in HCC development to further optimize therapeutic efficiency.

Among hallmarks in cancer formation, dependence on glycolysis is one of the important features in various kinds of cancer including HCC (5-9). In addition to glucose, cancer cells remodel the metabolism of other macromolecules, including amino acids $(5,10)$ and fatty acids $(11,12)$, to support neoplasia growth. Our laboratory has demonstrated that dysregulated lipid metabolism by long-chain acyl-CoA synthetase (ACSL) expression (13) and dysregulated amino acid metabolism by argininosuccinate lyase (ASL) expression (14) play important roles in cancer formation.

Among dysregulated amino acid metabolism, glutamine, serine, and glycine are reported to regulate cancer formation and are potential therapeutic targets $(10,15)$. In addition, decreased arginine production is frequently observed in HCC and melanoma. Therefore, these tumors are susceptible to arginine deprivation therapy $(15,16)$. Arginase or arginine deiminase has been reported to display effective 
anticancer potential in HCC and melanoma in vitro (17-24), in vivo $(17-19,21,24)$, and in clinical trials (25-28).

However, arginine deprivation therapy may also, like other cancer chemotherapies and targeted therapies $(29,30)$, confront the problem of drug resistance $(15,16)$. To this end, molecular mechanisms in arginine metabolic enzymemediated cancer formation and arginine deprivation therapy deserve further elucidation.

Our laboratory previously identified that knockdown of the arginine metabolic enzyme ASL inhibits HCC formation in part through reduction of cyclin A2 (14). In this study, we further studied the mechanism by which ASL regulates cyclin A2 protein level. We found that ASL directly interacted with cyclin A2 in the cytoplasm of HCC cells. Mutant ASL which is devoid of arginine metabolic activity retained the ability to interact with cyclin A2 and promoted anchorageindependent growth, suggesting ASL/cyclin A2 interaction may be important for tumor growth. Furthermore, ASL overexpression modulated liver cancer progression regarding drug resistance especially to arginine deprivation therapy, with potential therapeutics counteracting above phenomenon being identified with bioinformatics analysis, which may provide an opportunity for improvement of treatment efficiency.

\section{Materials and methods}

Cell lines. Human HCC cell line Huh7 was kindly provided by I.J. Su at National Health Research Institute. Huh7 and another human HCC cell line HepG2 were cultured in DMEM media containing 10\% FBS (Biological Industries, Beit Haemek, Israel) and $1 \%$ penicillin-streptomycin. Cells were kept in incubator at $37^{\circ} \mathrm{C}$ and $5 \% \mathrm{CO}_{2}$. shASL-Huh7 and shASL-HepG2 were established as previously described (14).

Chemicals, reagents, plasmids, and antibodies. Gelatin, bovine serum albumin (BSA), 5-FU, cisplatin, and sorafenib were purchased from Sigma (St. Louis, MO, USA). Micro$\mathrm{BCA}^{\mathrm{TM}}$ protein assay reagent kit was purchased from Pierce (Woburn, MA, USA). DMEM and antibiotic mixture were purchased from Invitrogen (Carlsbad, CA, USA). Turbofect transfection reagent was purchased from Fermentas (Glen Burnie, MD, USA). Plasmids of ASL-Myc and cyclin A2-HA were as described previously (14). Antibodies against ASL (GTX113629; polyclonal; rabbit anti-human; GeneTex,Hsinchu, Taiwan), Myc (51-1485GR; monoclonal; mouse anti-Myc; BD Biosciences, Franklin Lakes, NJ, USA), Cyclin A2 (sc-596; polyclonal; rabbit anti-human; Santa Cruz Biotechnology, Dallas, TX, USA), HA (11867431001; monoclonal; rat anti-HA; Roche, Basel, Switzerland), HA (MMS-101P; monoclonal; mouse anti-HA; Covance, Princeton, NJ, USA), GAPDH (GTX100118; polyclonal; rabbit anti-human; GeneTex), Lamin A/C (ab108595; monoclonal; rabbit anti-human; Abcam, Cambridge, UK), GFP (GTX628528; monoclonal; mouse anti-GFP; GeneTex), and HALO (G9211; monoclonal; mouse anti-HALO; Promega, Sunnyvale, CA, USA) were used in western blotting (all 1:2000 except 1:1000 for ASL and 1:5000 for GAPDH), immunofluorescence (all 1:100), or co-immunoprecipitation. ADI-PEG was kindly provided by Polaris Pharmaceuticals (San Diego, CA, USA).
Immunofluorescence and confocal microscopy. Cancer cells $\left(5 \times 10^{4}\right)$ were seeded onto 6-well plates containing cover glasses pre-coated with $0.1 \%$ gelatin and cultured overnight. Cells were then washed with PBS and fixed with $3.7 \%$ paraformaldehyde. Following wash with $0.1 \mathrm{M}$ glycine, cells were treated with permeabilization/blocking buffer (2\% FBS, $0.4 \%$ Triton X-100 in PBS). After washing with wash/staining buffer (0.2\% BSA, $0.2 \%$ Triton X-100 in PBS), cells were probed with primary antibodies overnight at $4^{\circ} \mathrm{C}$. Following washing, cells were probed with secondary antibodies at room temperature for $1 \mathrm{~h}$ in the dark. Cells were then stained with DAPI after washing. Subcellular localization and co-localization of ASL and/or cyclin A2 were then analyzed by confocal microscope Olympus FV1000MPE (Olympus, Tokyo, Japan) with 63X oil immersion objective. Fluorescent images were taken in sequential scanning mode (instead of simultaneous one) to avoid non-specific fluorescent signal during image acquisition. $\mathrm{IgG}$ was used as a negative control.

Nuclear-cytosolic fractionation. Cells were subjected to nuclear-cytosolic fractionation according to the manufacturer's instructions (Thermo Fisher Scientific, Waltham, MA, USA).

Western blotting. Cells were lysed in modified RIPA buffer with protease inhibitors and let stand on ice for $20 \mathrm{~min}$. After centrifugation at $15,000 \mathrm{xg}$ at $4^{\circ} \mathrm{C}$ for $10 \mathrm{~min}$, supernatant was harvested and protein concentration was assayed by micro-BCA protein assay reagent kit (Thermo Fisher Scientific). Samples with same amount of protein and $4 \mathrm{X}$ sample buffer were mixed, heated at $95^{\circ} \mathrm{C}$ for $5 \mathrm{~min}$, and subjected to electrophoresis. The protein was then transferred onto PVDF membrane (Millipore, Bedford, MA, USA) by Hoefer Semiphor Semi-Dry transfer unit (Amersham Pharmacia Biotech, Inc., San Francisco, CA, USA), and blocked in 5\% non-fat milk at room temperature for $1 \mathrm{~h}$. Membrane was then probed with primary antibody overnight at $4^{\circ} \mathrm{C}$. Following washing with $0.1 \%$ TBS-T, membrane was probed with secondary antibody at room temperature for $1 \mathrm{~h}$. After washing, the quantity of targets were identified by adding chemiluminescence reagent ECL (Millipore) onto membrane and luminescent intensity was recorded by BioSpectrum AC imaging system (UVP Inc., Upland, CA, USA).

Co-immunoprecipitation. Cells were lysed in immunoprecipitation buffer containing NP-40 or Triton X-100 with protease inhibitors and harvested as in western blotting or in nuclearcytosolic fractionation, and protein was quantified. Samples (5\%) were loaded in input lane. Remaining samples were added with $2 \mu \mathrm{g}$ primary antibody for immunoprecipitation at $4^{\circ} \mathrm{C}$ overnight with agitation. $30 \mu \mathrm{l}$ protein $\mathrm{G}$ magnetic beads (Millipore) or $100 \mu \mathrm{l}$ protein $\mathrm{G}$ agarose beads (Millipore) were then washed, mixed gently with samples, and incubated at $4^{\circ} \mathrm{C}$ for $2 \mathrm{~h}$ with agitation. Following washing, samples were eluted from beads by $4 \mathrm{X}$ sample buffer with heating at $95^{\circ} \mathrm{C}$ for $5 \mathrm{~min}$, and subjected to electrophoresis.

Site-directed mutagenesis and polymerase chain reaction (PCR).Plasmids of ASL-Myc and cyclin A2-HA were subjected to site-directed mutagenesis using QuikChange Site-Directed Mutagenesis kit (Agilent Technologies, Inc., Santa Clara, CA, USA), HiFi HotStart PCR kit (Kapa Biosystems, Wilmington, 
A

\begin{tabular}{l|l} 
& MAPRE1 \\
& IgG \\
& HA \\
IP & IB: (ASL)-Myc \\
& IB: (CCNA2)-HA \\
Input & IB: (ASL)-Myc \\
& IB: (CCNA2)-HA
\end{tabular}

B

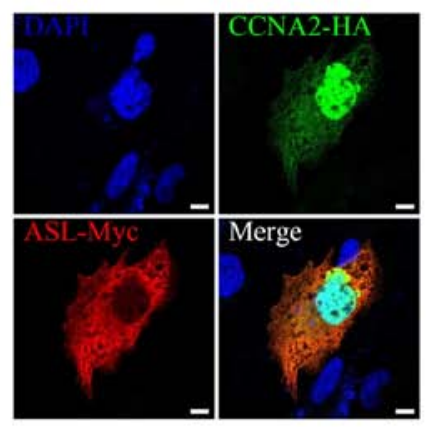

C

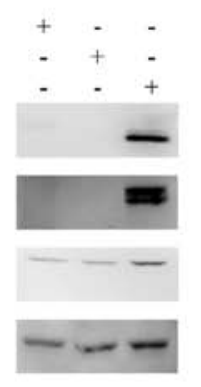

Vector

$\mathrm{ASL}+\mathrm{CCNA} 2$

IgG

GFP

SGK1

\begin{tabular}{l|l} 
IP & IB: (ASL)-HALO \\
& IB: (CCNA2)-GFP
\end{tabular}

\begin{tabular}{l|l} 
Input & IB: (ASL)-HALO \\
IB: (CCNA2)-GFP
\end{tabular}

D

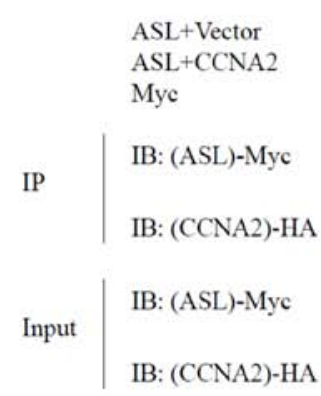

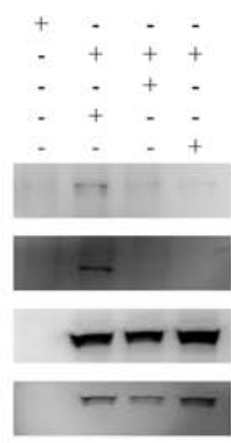

F

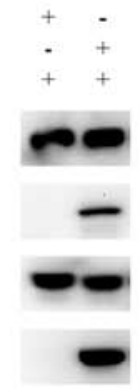

E

Vector

ASL+CCNA2

Cytosolic fraction

Nuclear fraction

IB: (ASL)-Myc

IB: (CCNA2)-HA

IB: GAPDH

IB: Lamin A/C

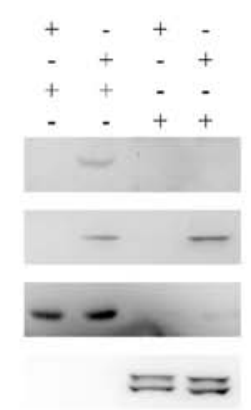

$\mathrm{ASL}+\mathrm{CCNA} 2$

Cytosolic fraction

Nuclear fraction

IgG

HA

\begin{tabular}{l|l} 
IP & IB: (ASL)-Myc \\
& IB: (CCNA2)-HA
\end{tabular}

IB: (ASL)-Myc

IB: (CCNA2)-HA

Input

IB: GAPDH

IB: Lamin A/C

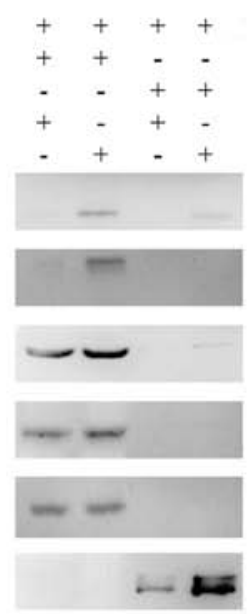

Figure 1. ASL interacted and colocalized with cyclin A2 in the cytosol of HCC cells. Huh7 was cotransfected with ASL-Myc and cyclin A2-HA, and (A) the interaction between ASL-Myc and cyclin A2-HA was determined by immunoprecipitation of HA and subsequent detection of Myc expression by western blotting, with anti-rat antibody MAPRE1 serving as negative control; (B) the subcellular localization and colocalization of ASL-Myc and cyclin A2-HA were determined by immunofluorescence and confocal microscope analysis, scale bar $10 \mu \mathrm{m}$; (C) 293 cells were cotransfected with vectors or ASL-HALO and cyclin A2-GFP, and the interaction between ASL-HALO and cyclin A2-GFP was determined by immunoprecipitation of GFP and subsequent detection of HALO expression by western blotting, with anti-mouse antibody SGK1 serving as negative control. Results are from three independent experiments; (D) 293 cells were cotransfected with ASL-Myc and cyclin A2-HA or its vector control, and the interaction between ASL-Myc and cyclin A2-HA was determined by immunoprecipitation of Myc and subsequent detection of HA expression by western blotting; (E) the nuclear-cytosolic distribution of ASL-Myc and cyclin A2-HA was determined by nuclear-cytosolic fractionation followed by western blotting; (F) the subcellular localization of ASL/cyclin A2 interaction was determined by nuclear-cytosolic fractionation followed by immunoprecipitation of HA and subsequent detection of Myc expression by western blotting. Results are from three independent experiments.

MA, USA), and Applied Biosystems 2720 Thermal Cycler (Applied Biosystems, Foster City, CA, USA) according to the manufacturer's instructions to establish loss-of-enzymatic activity mutant ASL-Myc and cyclin A2-HA. Primers for sitedirected mutagenesis were purchase from MDBio, Inc. (Taipei, Taiwan) and listed below: ASL G532A: (forward) 5'-ATTCT GAGCCACGCCATGGCACTGACCCGAG-3', (reverse) 5'-CTCGGGTCAGTGCCATGGCGTGGCTCAGAAT-3'; ASL A857G: (forward) 5'-GCAGCCTGATGCCCCGGAAG AAAAACCCCG A-3', (reverse) 5'-TCGGGGTTTTTCTTC CGGGGCATCAGGCTGC-3'; CCNA2 M210A: (forward) 5'-AGCCAGACATCACTAACAGTGCGAGAGCTATCCTC GTGGACT-3', (reverse) 5'-AGTCCACGAGGATAGCTCTC GCACTGTTAGTGATGTCTGGCT-3'; CCNA2 L214A: (forward) 5'-ACTAACAGTGCGAGAGCTATCGCCG TGGACTGGTTAGTTGAAGTA-3', (reverse) 5'-TACTT CAACTAACCAGTCCACGGCGATAGCTCTCGCACTG TAGCTCTCAT-3'. Final concentration for reagents for sitedirected mutagenesis were described as below: 1X KAPA HiFi buffer (GC), $0.3 \mathrm{mM}$ KAPA dNTP Mix, $0.3 \mu \mathrm{M}$ forward/ reverse primer, $50 \mathrm{ng}$ cDNA, $1 \mathrm{U} / \mu 1 \mathrm{KAPA}$ HiFi HotStart DNA polymerase in $50 \mu \mathrm{l}$ reaction. PCR for site-directed mutagenesis is described below: at $95^{\circ} \mathrm{C}$ for $30 \mathrm{sec}, 1 \mathrm{cycle} ; 95^{\circ} \mathrm{C}$ for $30 \mathrm{sec}, 55^{\circ} \mathrm{C}$ for $1 \mathrm{~min}, 68^{\circ} \mathrm{C}$ for $1 \mathrm{~min} 30 \mathrm{sec}, 18$ cycles.

RNA interference. The shASL plasmid-carrying bacterial clones and corresponding pseudo lentivirus were obtained from RNAi core facility (Academia Sinica, Taipei, Taiwan) and the shRNA targets 3' UTR sequences: 5'-AGGAGGCTGCTGTGTGTTT-3'. Cells were infected with pseudo lentivirus against ASL or luciferase vector control, selected by $2 \mu \mathrm{g} / \mathrm{ml}$ puromycin for 2 days, and subjected to further experiments.

Anchorage-dependent growth by colony formation assay. Cancer cells $\left(1 \times 10^{3}\right)$ were seeded onto 6 -well plates and cultured for 9 days to assay the anchorage-dependent growth ability of cancer cells. The number of colonies was identified by $2 \%$ methyl blue staining, counted and analyzed.

Anchorage-independent growth by soft agar growth assay. Cells $\left(5 \times 10^{3}\right)$ were seeded into $1 \mathrm{ml} 0.3 \%$ agar-containing 

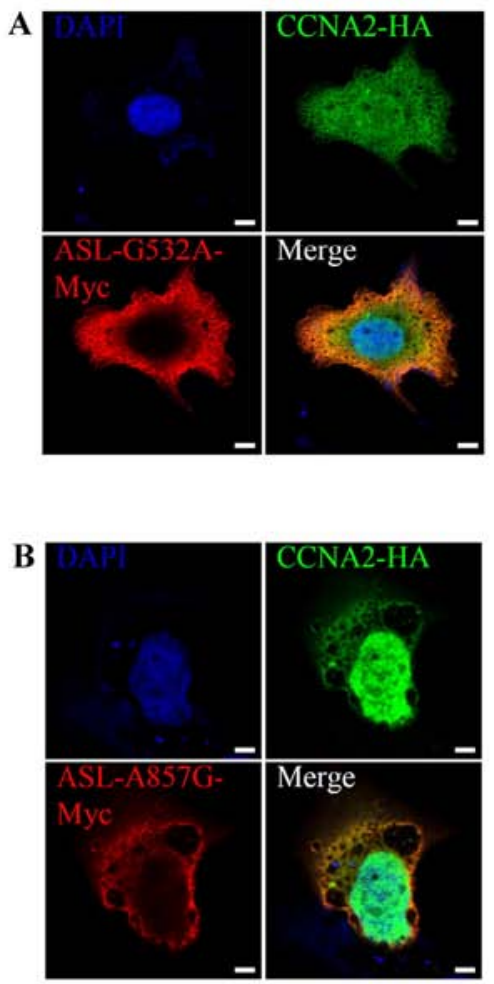
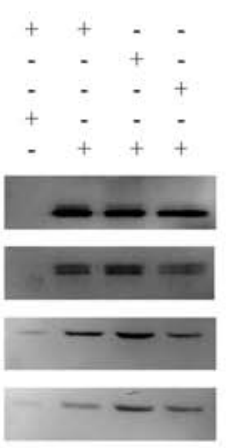

D

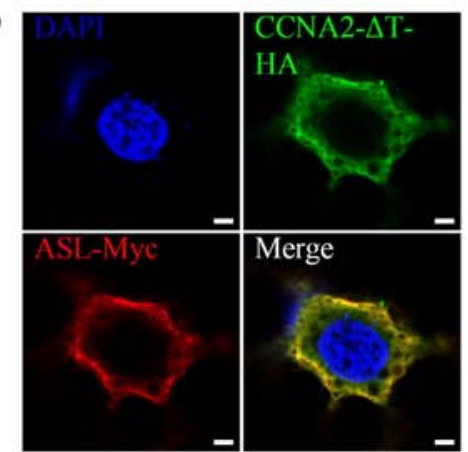

E

\section{$\mathrm{ASL}+\mathrm{CCNA} 2$}

$A S L+C C N A 2-\Delta T$

IgG

HA

\begin{tabular}{l|l} 
IP & IB: (ASL)-Myc \\
& IB: (CCNA2)-HA
\end{tabular}

IB: (ASL)-Myc

Input

IB: (CCNA2)-HA

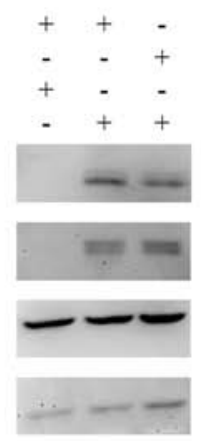

Figure 2. Mutant ASL or mutant cyclin A2 retain the ASL/cyclin A2 interaction ability in HCC cells. Huh7 was cotransfected with wild-type, G532A mutant or A857G mutant ASL and wild-type cyclin A2. The colocalization (A and B) as well as interaction (C) were determined by confocal microscope analysis or immunoprecipitation of HA and subsequent detection of Myc expression by western blotting, respectively. Huh7 was cotransfected with wild-type ASL and mutant cyclin A2 containing M210A, L214A, W217A triple mutation ( $\Delta \mathrm{T})$. The colocalization (D) as well as interaction (E) were determined similarly. Scale bar, $10 \mu \mathrm{m}$. Results are from three independent experiments.

medium and then onto the $0.6 \%$ agar-covered 6 -well plates and cultured for 14 days to assay the anchorage-independent growth ability of cancer cells. The number of colonies was identified by $0.05 \%$ crystal violet staining, counted and analyzed.

Microarray analysis. Total RNA of Huh7 and shASL-Huh7 stable transfectants were extracted using TRIzol reagent (MDBio, Inc., Taiwan). The microarray analysis was performed using Whole Human Genome Oligo Microarray kit (4x44 K) (Agilent Technologies, Inc.) by Welgene Biotech, Co., Ltd. (Taipei, Taiwan).

Metacore bioinformatics analysis. The pathway analysis database Metacore (https://portal.genego.com/cgi/data_manager. cgi\#) (31) was applied with default setting. The analyses with false discovery rate (FDR) $<0.05$ were displayed as pathway maps (pathways from literature consensus).

Connectivity Map bioinformatics analysis. The gene-drug interaction database Connectivity Map (https://www.broad institute.org/cmap/) $(32,33)$ was applied with default setting. The differentially expressed genes in shASL-Huh7 were divided into top 500 upregulated and downregulated groups and uploaded to Connectivity Map database for analysis of potential therapeutics with gene expression signature mimicking ASL knockdown in Huh7.
Statistical analysis. All statistical analyses were performed with the GraphPad Prism version 5 (GraphPad Software, San Diego, CA, USA). All error bars of the figures represent SEM. Student's t-test and two-way ANOVA followed by Bonferroni post-test were used for analysis of difference between each experimental group. P-value of $<0.05$ was considered to be significant.

\section{Results}

ASL colocalizes and interacts with cyclin A2 in the cytoplasm of HCC cells. We have demonstrated that downregulation of ASL by shRNA inhibits tumor growth which is in part mediated through downregulating cyclin A2 expression. The reduction of cyclin A2 is probably regulated at the protein level because cyclin A2 mRNA was not altered (14). Based on this information, we hypothesized that ASL might directly interact with cyclin A2 and regulate its protein expression. We cotransfected ASL-Myc and cyclin A2-HA into Huh7 and investigated their colocalization and interaction by immunofluorescence and immunoprecipitation, respectively. ASL interacted (Fig. 1A) and colocalized (Fig. 1B) with cyclin A2 in Huh7 liver cancer cells. Double-band was observed in immunoprecipitation of HA-cyclin A2, but not in the input of HA-cyclin A2. As cyclin A2 is easily degraded, we speculated that the double bands may be resulted from the degradation of cyclin A2 during immuno- 
A shLuc

shASL

ASL

ASL-G532A

ASL-A857G

CCNA2

CCNA2- $\Delta \mathrm{T}$

IB: ASL

IB: CCNA2

IB: GAPDH

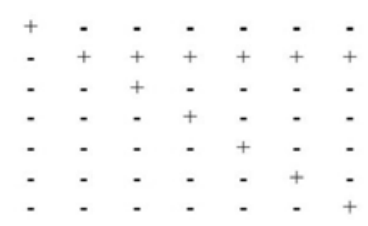

$\longrightarrow$

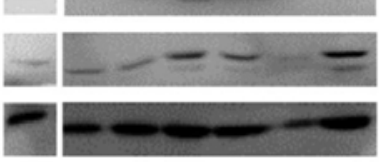

C

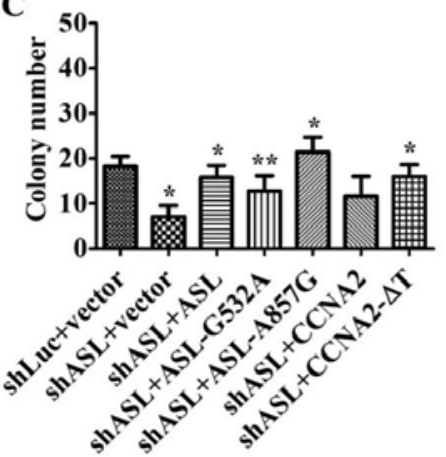

D

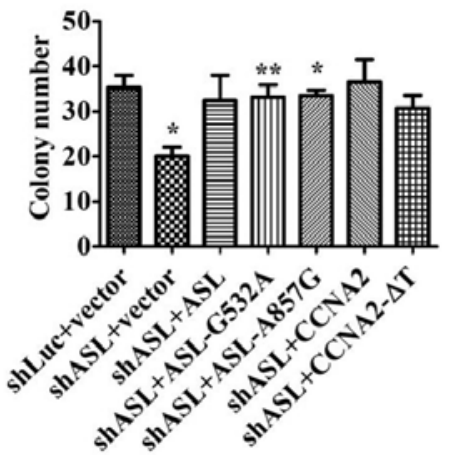

E

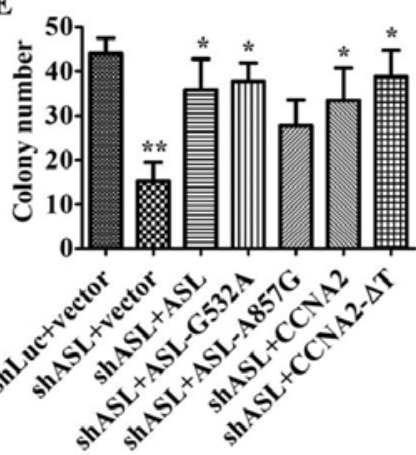

F

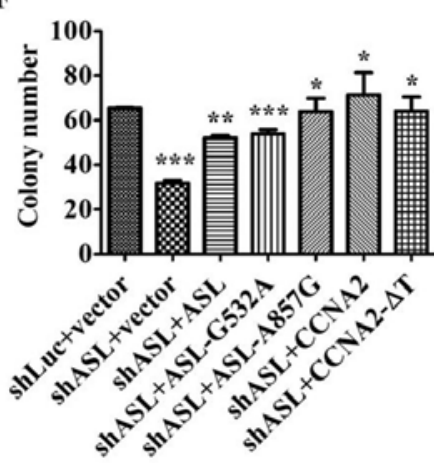

Figure 3. Mutant ASL or cyclin A2 retained the ability to restore cell growth repressed by shASL in hepatocarcinoma cells. shASL-Huh7 (A, C and E) or shASL-HepG2 (B, D and F) was transfected with wild-type or mutant of ASL or cyclin A2, and the expression of the above molecules was determined by western blotting (A and B) from the same set of experiments on the same gel. Their effects on anchorage-dependent and -independent growth were determined by colony formation assay $(\mathrm{C}$ and $\mathrm{D})$ and soft agar growth assay ( $\mathrm{E}$ and $\mathrm{F})$, respectively. Results were from three independent experiments, and error bars represent SEM, with the statistical difference between the anchorage-dependent and -independent growth ability of above mentioned overexpression clones and control clone was examined with Student's t-test $\left({ }^{*} \mathrm{P}<0.05 ;{ }^{* *} \mathrm{P}<0.01,{ }^{* * * *} \mathrm{P}<0.001\right)$.

precipitation. To avoid IgG heavy chain interruption during co-immunoprecipitation, we applied EasyBlot IgG HRP secondary antibody and observed no obvious signal resulting from IgG heavy chain. This interaction is also observed by coexpression of exogenous ASL-HALO and cyclin A2-GFP in 293 cells (Fig. 1C). In addition, we have performed the experiments with ASL-Myc and cyclin A2-HA or HA-vector control and we observed no interaction between ASL-Myc and vector control, supporting that the specific interaction between ASL and cyclin A2 indeed occurred under co-overexpression (Fig. 1D). Altogether, the arginine metabolic enzyme ASL co-localized and interacted with cell cycle regulator cyclin A2.

To further identify the subcellular localization of ASL/cyclin A2 interaction, ASL-Myc and cyclin A2-HA were cotransfected into Huh7 and nuclear-cytosolic fractionation without or with subsequent immunoprecipitation. ASL is mainly localized in the cytosol of Huh7, and cyclin A2 is located in both cytosol and the nucleus (Fig. 1E), suggesting the subcellular localization for ASL/cyclin A2 interaction was in the cytosol. Furthermore, the interaction between ASL and cyclin A2 was detected in the cytosolic fraction of Huh7 with immunoprecipitation (Fig. 1F). This result indicated that the interaction between ASL and cyclin A2 mainly occurs in the cytosol of liver cancer cells.
Loss-of-enzymatic activity mutant ASL retains the ability to interact with cyclin A2 and promotes cell growth. In order to determine whether the metabolic enzymatic activity of ASL is required for the interaction, we constructed mutant ASL-Myc without enzymatic activity. Previous results indicated that genomic mutations in ASL leading to G532A or A857G amino acid substitution were observed in patients with ASL function deficiency (34). On the other hand, cyclin A2 containing M210A, L214A, W217A triple mutation ( $\Delta$ Triple) is unable to influence cyclin-dependent kinase activity in human $(35,36)$. We then investigated the interaction between wild-type ASL and mutant cyclin A2 or mutant ASL and wild-type cyclin A2. Immunofluorescence and immunoprecipitation analysis revealed that mutant ASL or cyclin A2 still retained the ability to colocalize (Fig. 2A, B and D) and interact (Fig. 2C and E) with wild-type interaction partner. This result revealed that ASL enzymatic activity is not essential for the interaction with cyclin A2.

To elucidate the importance of the interaction between ASL and cyclin A2 in liver cancer promotion, we delivered mutant ASL or mutant cyclin A2 into Huh7 ASL shRNA stable transfectants, and observed whether they could restore the growth inhibition by ASL shRNA. Both wild-type and lossof-enzymatic activity ASL or cyclin A2 were able to restore the growth of shASL-Huh7 stable transfectants in colony formation assay and soft agar growth assay in vitro (Fig. 3). 
A $\begin{array}{ll}\text { Vector } & + \\ \text { ASL-Myc } & - \\ \text { IB: (ASL)-Myc } & \\ \text { IB: GAPDH } & \end{array}$

C
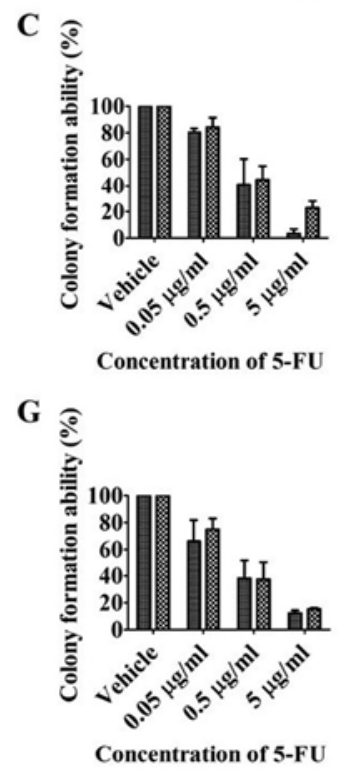

K

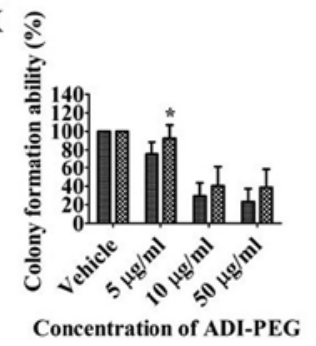

B Vector

ASL-Myc

IB: (ASL)-Myc

IB: GAPDH

D

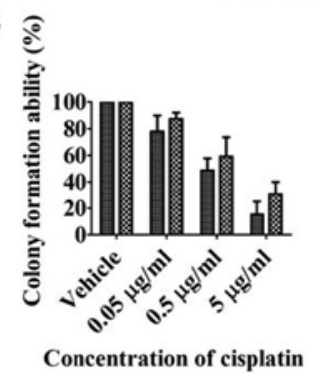

H

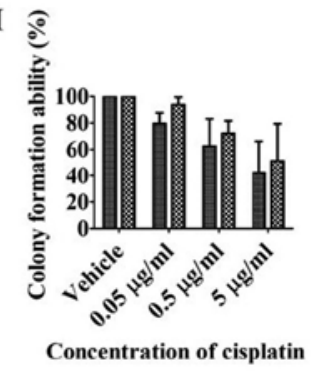

L

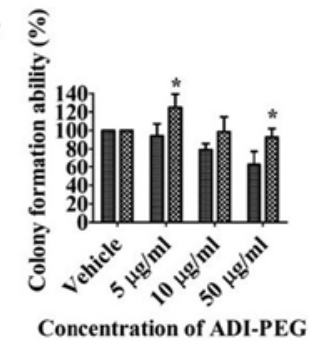

E

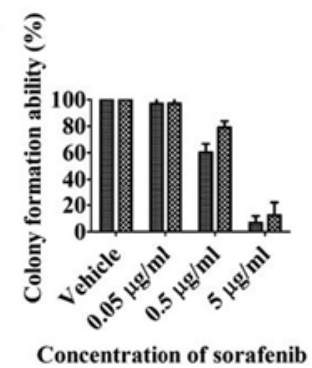

I

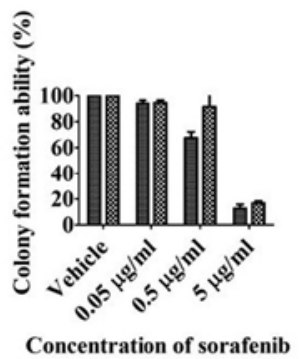

F

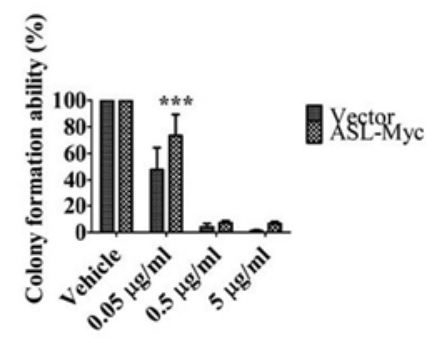

Concentration of ADI-PEG

$\mathbf{J}$

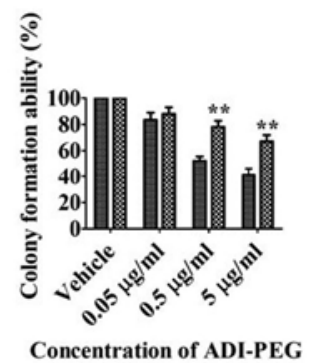

Figure 4. ASL overexpression promotes drug resistance against arginine deprivation therapy. Huh7 (A, C, D, E, F and K) or HepG2 (B, G, H, I, J and L) was transfected with ASL-Myc or vector control, and the expression was determined by western blotting (A and B). The growth of cells in the presence of anticancer drugs including the chemotherapies with 5-FU (C and G) and cisplatin (D and H), targeted therapy sorafenib (E and I), and arginine deprivation therapy ADI-PEG (F, J, K and L) was determined by colony formation assay (C-J) and soft agar growth assay (K and L). Results are from three independent experiments, and error bars represent SEM, the statistical difference between the anchorage-dependent and -independent growth ability of above mentioned overexpression clones and control clone was examined with two-way ANOVA followed by Bonferroni post-test $\left({ }^{*} \mathrm{P}<0.05 ;{ }^{* * *} \mathrm{P}<0.01,{ }^{* * * * *} \mathrm{P}<0.001\right)$.

Therefore, loss-of-enzymatic activity ASL or cyclin A2 still maintained the ability to promote anchorage-dependent growth and anchorage-independent growth in liver cancer cells. Since these mutants retained the ability to form the ASL/cyclin A2 complex, these results imply that the novel ASL/cyclin A2 interaction may play a role in mediating cell growth (Figs. 2 and 3).

ASL overexpression promotes drug resistance to arginine deprivation therapy. To identify the impact of ASL on tumor progression in terms of drug resistance, we transfected ASL-Myc or vector control into Huh7 and HepG2 and investigated their resistance toward chemotherapies with 5-FU, cisplatin, multiple tyrosine kinase sorafenib (4), and arginine deprivation therapy ADI-PEG (Fig. 4A and B). Overall, ectopic expression of ASL resulted in an increased tendency of resistance toward these anticancer drugs in colony formation assay although the difference did not reach statistical significance (Fig. 4C, D, E, G, H and I). Importantly, ASL overexpression conferred drug resistance to arginine deprivation therapy
ADI-PEG with anchorage-dependent growth (Fig. 4F and J) and anchorage-independent growth (Fig. 4K and L).

Bioinformatics analyses reveal potential therapeutics in ASL-regulated HCC formation. Since ASL played an important role in liver tumor formation, we performed microarray gene expression analysis on the comparison of Huh7 cells and ASL shRNA stable transfectants. The downregulated genes by ASL shRNA in Huh7 cells were selected, and uploaded to Metacore bioinformatics database for identification of important pathways with its default setting. The analyses were displayed as pathway maps. The gene expression signature resulted from ASL knockdown in Huh7 was associated with cell cycle progression, cytoskeleton remodeling, apoptosis, and immune responses (Table I).

To further identify therapeutic options for ASL-regulated HCC formation, we applied the gene expression signature of ASL-knockdown in Huh7 cells in Connectivity Map bioinformatics database $(32,33)$ with default setting. The gene expression signature was associated with the treatment 
Table I. Bioinformatics analysis with Metacore database revealed important pathways in ASL-regulated HCC formation.

\begin{tabular}{|c|c|c|}
\hline Rank & Maps & FDR \\
\hline 1 & Transport_Clathrin-coated vesicle cycle & 4.287E-15 \\
\hline 2 & Cytoskeleton remodeling_TGF, WNT and cytoskeletal remodeling & $4.287 \mathrm{E}-15$ \\
\hline 3 & Cell cycle_Start of DNA replication in early $\mathrm{S}$ phase & $3.031 \mathrm{E}-11$ \\
\hline 4 & DNA damage_ATM/ATR regulation of G1/S checkpoint & $5.234 \mathrm{E}-10$ \\
\hline 5 & Cell cycle_Influence of Ras and Rho proteins on G1/S Transition & $6.190 \mathrm{E}-10$ \\
\hline 6 & Cytoskeleton remodeling_Cytoskeleton remodeling & 8.999E-10 \\
\hline 7 & DNA damage_Role of Brca1 and Brca2 in DNA repair & 2.617E-09 \\
\hline 8 & Apoptosis and survival_Endoplasmic reticulum stress response pathway & $3.117 \mathrm{E}-09$ \\
\hline 9 & Development_Differentiation of white adipocytes & $1.489 \mathrm{E}-08$ \\
\hline 10 & Immune response_Role of PKR in stress-induced antiviral cell response & $1.489 \mathrm{E}-08$ \\
\hline 11 & Transcription_Epigenetic regulation of gene expression & $1.489 \mathrm{E}-08$ \\
\hline 12 & Immune response_IL-1 signaling pathway & $1.843 \mathrm{E}-08$ \\
\hline 13 & Development_Regulation of telomere length and cellular immortalization & $1.843 \mathrm{E}-08$ \\
\hline 14 & Transcription_Sirtuin6 regulation and functions & $2.722 \mathrm{E}-08$ \\
\hline 15 & Apoptosis and survival_TNFR1 signaling pathway & $4.026 \mathrm{E}-08$ \\
\hline 16 & G-protein signaling_RhoA regulation pathway & $4.062 \mathrm{E}-08$ \\
\hline 17 & Apoptosis and survival_Role of PKR in stress-induced apoptosis & $4.952 \mathrm{E}-08$ \\
\hline 18 & Translation_Regulation of EIF4F activity & $4.952 \mathrm{E}-08$ \\
\hline 19 & Apoptosis and survival_Role of IAP-proteins in apoptosis & $6.565 \mathrm{E}-08$ \\
\hline 20 & Oxidative phosphorylation & $7.756 \mathrm{E}-08$ \\
\hline 21 & Apoptosis and survival_FAS signaling cascades & 8.099E-08 \\
\hline 22 & IGF family signaling in colorectal cancer & $9.132 \mathrm{E}-08$ \\
\hline 23 & Cell cycle_Transition and termination of DNA replication & $9.906 \mathrm{E}-08$ \\
\hline 24 & Regulation of degradation of deltaF508-CFTR in CF & $1.317 \mathrm{E}-07$ \\
\hline 25 & DNA damage_Brca1 as a transcription regulator & $1.317 \mathrm{E}-07$ \\
\hline 26 & Signal transduction_Additional pathways of NF- $\mathrm{NB}$ activation (in the nucleus) & $1.317 \mathrm{E}-07$ \\
\hline 27 & Transcription_Transcription regulation of aminoacid metabolism & $1.433 \mathrm{E}-07$ \\
\hline 28 & Signal transduction_AKT signaling & $1.495 \mathrm{E}-07$ \\
\hline 29 & Development_WNT signaling pathway. Part 2 & $1.687 \mathrm{E}-07$ \\
\hline 30 & Development_NOTCH1-mediated pathway for NF- $\kappa \mathrm{B}$ activity modulation & $2.002 \mathrm{E}-07$ \\
\hline 31 & Cell cycle_The metaphase checkpoint & $2.346 \mathrm{E}-07$ \\
\hline 32 & Role of Tissue factor-induced Thrombin signaling in cancerogenesis & $2.416 \mathrm{E}-07$ \\
\hline 33 & Cell cycle_Role of SCF complex in cell cycle regulation & $2.746 \mathrm{E}-07$ \\
\hline 34 & Proteolysis_Putative SUMO-1 pathway & $2.746 \mathrm{E}-07$ \\
\hline 35 & Apoptosis and survival_Apoptotic TNF-family pathways & $2.920 \mathrm{E}-07$ \\
\hline 36 & Development_IGF-1 receptor signaling & $3.075 \mathrm{E}-07$ \\
\hline 37 & Development_TGF- $\beta$ receptor signaling & $3.075 \mathrm{E}-07$ \\
\hline 38 & Cell cycle_ESR1 regulation of G1/S transition & 4.112E-07 \\
\hline 39 & Cell cycle_Cell cycle (generic schema) & $4.236 \mathrm{E}-07$ \\
\hline 40 & Cell cycle_Chromosome condensation in prometaphase & $4.236 \mathrm{E}-07$ \\
\hline 41 & DNA damage_ATM/ATR regulation of G2/M checkpoint & $4.236 \mathrm{E}-07$ \\
\hline 42 & Cell cycle_Regulation of G1/S transition (part 2) & $4.236 \mathrm{E}-07$ \\
\hline 43 & Apoptosis and survival_DNA-damage-induced apoptosis & $4.314 \mathrm{E}-07$ \\
\hline 44 & Transport_RAN regulation pathway & $5.083 \mathrm{E}-07$ \\
\hline 45 & Signal transduction_Additional pathways of NF- $\kappa \mathrm{B}$ activation (in the cytoplasm) & $5.433 \mathrm{E}-07$ \\
\hline 46 & IL-6 signaling in multiple myeloma & $5.433 \mathrm{E}-07$ \\
\hline 47 & Signal transduction_NF- $\kappa$ B activation pathways & $5.433 \mathrm{E}-07$ \\
\hline 48 & Oxidative stress_Role of Sirtuin1 and PGC1- $\alpha$ in activation of antioxidant defense system & $8.745 \mathrm{E}-07$ \\
\hline 49 & Apoptosis and survival_Caspase cascade & $9.568 \mathrm{E}-07$ \\
\hline 50 & Cell cycle_Initiation of mitosis & $9.751 \mathrm{E}-07$ \\
\hline
\end{tabular}

The genes with downregulated expression in shASL-Huh7 were previously subjected to microarray analysis and the result together with the signal intensity was uploaded to Metacore database and analyzed for pathway maps (pathways from literature consensus) in shASL-reduced HCC formation, ranked according to statistical significance and exported as table. FDR, false discovery rate. 


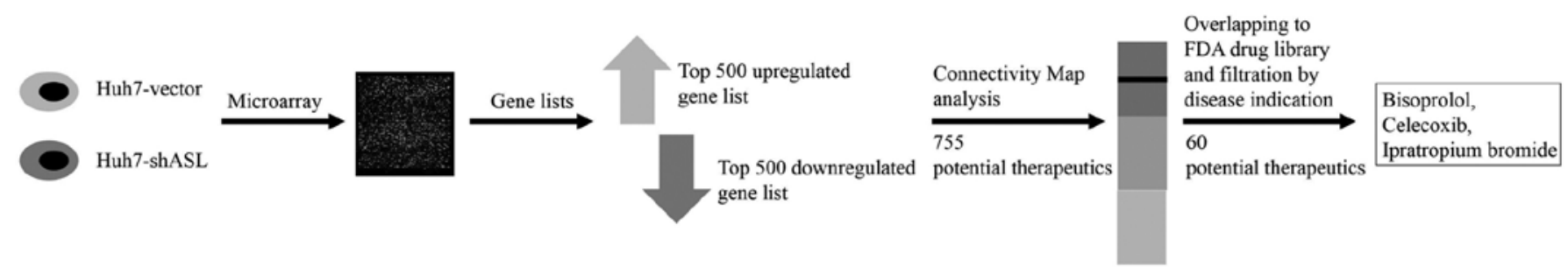

Figure 5. Flow chart of bioinformatics analysis with Connectivity Map database revealing potential therapeutics for ASL-regulated HCC formation. The gene expression signature of ASL knockdown in Huh7 was reorganized as gene lists of top 500 upregulated and downregulated groups and uploaded to Connectivity Map database, and 755 potential therapeutics with such signature were identified, which were further narrowed down by overlapping to the FDA drug library and exclusion of known indication in neural and cardiovascular diseases, with the final potential therapeutics being bisoprolol, celecoxib, and ipratropium bromide.

on cancer cells of 755 drugs in Connectivity Map analysis, in which 60 drugs overlapped with the FDA drug library available for testing the drug repurposing. Since drugs used in neural and cardiovascular diseases may confer stronger side effects to patients, we further excluded drugs with indications in these two fields. The potential novel therapeutics were narrowed down to i) bisoprolol, a $\beta 1$-adrenergic receptor blocker, ii) celecoxib, a COX-2 selective non-steroidal antiinflammatory drug (NSAID), and iii) ipratropium bromide, an anticholinergic function regulator (Fig. 5). This result pointed out the potential therapeutics for ASL-regulated HCC formation. These FDA-approved drugs deserve further elucidation on the combination with current cancer therapy for HCC.

\section{Discussion}

In the present study, we demonstrated that ASL interacted with cyclin A2 in the cytosol of HCC cells and the interaction might be important for tumor growth. We further found that ASL overexpression conferred drug resistance especially against arginine deprivation therapy. Bioinformatics analysis revealed that several drugs might be used to target ASL-overexpressing liver tumors.

The interaction was detected by the exogenous expression of ASL and cyclin A2. We have tried to determine the interaction between endogenous proteins, but was unable to detect the interaction between endogenous ASL and cyclin A2. This could be due to limited expression of ASL and cyclin A2 or the antibody binding site may interfere with the binding between ASL and cyclin A2. Therefore, we have used two different types of tagged exogenous ASL and cyclin A2 for IP in two different cell lines to sustain our conclusion (Fig. 1). It is interesting to note that the interaction between ASL and cyclin A2 is mainly localized in the cytosolic fraction. We further examined whether cytosolic cyclin A2 is attenuated by ASL shRNA. Nuclear/cytosolic fraction clearly demonstrated that cytosolic cyclin A2 is downregulated specifically. In contrast, nuclear cyclin A2 remained constant (data not shown). This result supports the notion that cytosolic ASL/cyclin A2 interaction influences its protein level. It should be noted that cyclin A2 may shuttle between nucleus and cytosol (37). The distribution of cyclin A2 is dependent on the cell cycle, and may be variable between different experiments. Jackman and coworkers (37) applied nuclear export assay to specifically address whether cyclin A could export from nucleus to cytosol spontaneously since it does not have nuclear localization signal (NLS), and found out that cyclin A indeed shuttled between nucleus and cytosol. This suggests that it is the interaction with other proteins, such as other cell cycle regulators containing NLS, that determines the subcellular localization of cyclin A. This phenomenon may also occur in the present study since upregulated ASL expression during $\mathrm{HCC}$ formation may interact, protect, and retain cyclin A2 in cytosol to execute biological functions in addition to the cell cycle regulator.

The interaction between wild-type and mutant forms of ASL and cyclin A2 is similar in co-immunoprecipitation (Fig. 2). The results indicate that the functional enzymatic mutation does not influence the interaction between ASL and cyclin A2. This observation supports our hypothesis that non-enzymatic function of ASL may influence cell cycle progression and tumor formation. Non-enzymatic functions of metabolic gene products have been reported to participate in cancer promotion and progression. For example, non-enzymatic function of heparanase promotes glioblastoma growth in vivo via activation of Akt signaling $(38,39)$. Non-enzymatic function of metabolic enzyme methylenetetrahydrofolate dehydrogenase/cyclohydrolase (MTHFD2) promotes colon cancer growth in vitro via association with DNA synthesis (40); non-enzymatic function of ATP-citrate lyase also enhances colon cancer growth in vitro by inhibiting AMPK signaling (41); non-enzymatic function of pyruvate kinase M2 (PKM2) promotes glioblastoma growth in vivo via activation of $\beta$-catenin signaling (42); non-enzymatic function of fructose-1,6-bisphosphatase 1 (FBP1) inhibits clear cell renal cell carcinoma progression in patients by reducing HIF function (43). The present study on ASL adds the importance of non-enzymatic functions of enzymes in regulating cancer formation $(40,44)$.

Based on the critical roles of ASL in drug resistance and cancer progression, the therapeutic potential targeting ASL overexpression deserves further attention. From the bioinformatics analysis with Connectivity Map database in the present study, bisoprolol, celecoxib, and ipratropium bromide were identified to be capable of counteracting ASL-regulated HCC formation. Our bioinformatics predictions are in accordance with a previous study on the targets of these drugs. These targets, including $\beta 1$-adrenergic receptor, COX-2, and cholinergic receptor, are involved in various kinds of cancer (45-48). 
In summary, we identified the cytosolic interaction between ASL and cyclin A2. This interaction may be important for liver cancer growth. Overexpression of ASL may be related with drug resistance especially for arginine deprivation therapy.

\section{Acknowledgements}

Computational analyses were supported by the system provided by the Bioinformatics Core at National Cheng Kung University, Tainan, Taiwan. This study was supported by the grant to M.-D.L. 101-2320-B-006-028-MY3 from Ministry of Science and Technology, Taiwan and NHRI-EX100-9927B1 from National Health Research Institute, Taiwan.

\section{References}

1. El-Serag HB and Rudolph KL: Hepatocellular carcinoma: Epidemiology and molecular carcinogenesis. Gastroenterology 132: 2557-2576, 2007.

2. Thorgeirsson SS and Grisham JW: Molecular pathogenesis of human hepatocellular carcinoma. Nat Genet 31: 339-346, 2002.

3. Bruix J, Sala M and Llovet JM: Chemoembolization for hepatocellular carcinoma. Gastroenterology 127 (Suppl 1): S179-S188, 2004.

4. Zhu AX: Development of sorafenib and other molecularly targeted agents in hepatocellular carcinoma. Cancer 112 250-259, 2008

5. Ward PS and Thompson CB: Metabolic reprogramming: A cancer hallmark even warburg did not anticipate. Cancer Cell 21: 297-308, 2012.

6. Pelicano H, Martin DS, Xu RH and Huang P: Glycolysis inhibition for anticancer treatment. Oncogene 25: 4633-4646, 2006.

7. Pavlova NN and Thompson CB: The emerging hallmarks of cancer metabolism. Cell Metab 23: 27-47, 2016.

8. Vander Heiden MG: Targeting cancer metabolism: A therapeutic window opens. Nat Rev Drug Discov 10: 671-684, 2011.

9. Tennant DA, Durán RV and Gottlieb E: Targeting metabolic transformation for cancer therapy. Nat Rev Cancer 10: 267-277, 2010.

10. Locasale JW: Serine, glycine and one-carbon units: Cancer metabolism in full circle. Nat Rev Cancer 13: 572-583, 2013.

11. Tirado-Vélez JM, Joumady I, Sáez-Benito A, Cózar-Castellano I and Perdomo G: Inhibition of fatty acid metabolism reduces human myeloma cells proliferation. PLoS One 7: e46484, 2012.

12. Currie E, Schulze A, Zechner R, Walther TC and Farese RV Jr: Cellular fatty acid metabolism and cancer. Cell Metab 18: 153-161, 2013.

13. Chang YS, Tsai CT, Huangfu CA, Huang WY, Lei HY, Lin CF Su IJ, Chang WT, Wu PH, Chen YT, et al: ACSL3 and GSK-3 $\beta$ are essential for lipid upregulation induced by endoplasmic reticulum stress in liver cells. J Cell Biochem 112: 881-893, 2011.

14. Huang HL, Hsu HP, Shieh SC, Chang YS, Chen WC, Cho CY, Teng CF, Su IJ, Hung WC and Lai MD: Attenuation of argininosuccinate lyase inhibits cancer growth via cyclin A2 and nitric oxide. Mol Cancer Ther 12: 2505-2516, 2013.

15. Delage B, Fennell DA, Nicholson L, McNeish I, Lemoine NR, Crook T and Szlosarek PW: Arginine deprivation and argininosuccinate synthetase expression in the treatment of cancer. Int J Cancer 126: 2762-2772, 2010.

16. Feun L, You M, Wu CJ, Kuo MT, Wangpaichitr M, Spector S and Savaraj N: Arginine deprivation as a targeted therapy for cancer. Curr Pharm Des 14: 1049-1057, 2008.

17. Ensor CM, Holtsberg FW, Bomalaski JS and Clark MA Pegylated arginine deiminase (ADI-SS PEG20,000 mw) inhibits human melanomas and hepatocellular carcinomas in vitro and in vivo. Cancer Res 62: 5443-5450, 2002.

18. Cheng PNM, Lam TL, Lam WM, Tsui SM, Cheng AW, Lo WH and Leung YC: Pegylated recombinant human arginase (rhArgpeg5,000 mw) inhibits the in vitro and in vivo proliferation of human hepatocellular carcinoma through arginine depletion. Cancer Res 67: 309-317, 2007.
19. Li YY, Wu C, Chen SM, Shah SS, Wangpaichitr M, Feun LG, Kuo MT, Suarez M, Prince J and Savaraj N: BRAF inhibitor resistance enhances vulnerability to arginine deprivation in melanoma. Oncotarget 7: 17665-17680, 2016.

20. Bobak Y, Kurlishchuk Y, Vynnytska-Myronovska B, Grydzuk O, Shuvayeva G, Redowicz MJ, Kunz-Schughart LA and Stasyk O: Arginine deprivation induces endoplasmic reticulum stress in human solid cancer cells. Int J Biochem Cell Biol 70: 29-38, 2016.

21. Miraki-Moud F, Ghazaly E, Ariza-McNaughton L, Hodby KA, Clear A, Anjos-Afonso F, Liapis K, Grantham M, Sohrabi F, Cavenagh J, et al: Arginine deprivation using pegylated arginine deiminase has activity against primary acute myeloid leukemia cells in vivo. Blood 125: 4060-4068, 2015.

22. Huang H-L, Chen W-C, Hsu H-P, Cho CY, Hung YH, Wang CY and Lai MD: Argininosuccinate lyase is a potential therapeutic target in breast cancer. Oncol Rep 34: 3131-3139, 2015.

23. Khoury O, Ghazale N, Stone E, El-Sibai M, Frankel AE and Abi-Habib RJ: Human recombinant arginase I (Co)-PEG5000 [HuArgI (Co)-PEG5000]-induced arginine depletion is selectively cytotoxic to human glioblastoma cells. J Neurooncol 122: 75-85, 2015.

24. Shan Y-S, Hsu H-P, Lai M-D, Yen MC, Chen WC, Fang JH, Weng TY and Chen YL: Argininosuccinate synthetase 1 suppression and arginine restriction inhibit cell migration in gastric cancer cell lines. Sci Rep 5: 9783, 2015.

25. Ott PA, Carvajal RD, Pandit-Taskar N, Jungbluth AA, Hoffman EW, Wu BW, Bomalaski JS, Venhaus R, Pan L, Old LJ, et al: Phase I/II study of pegylated arginine deiminase (ADI-PEG 20) in patients with advanced melanoma. Invest New Drugs 31: 425-434, 2013.

26. Yang TS, Lu SN, Chao Y, Sheen IS, Lin CC, Wang TE, Chen SC, Wang JH, Liao LY, Thomson JA, et al: A randomised phase II study of pegylated arginine deiminase (ADI-PEG 20) in Asian advanced hepatocellular carcinoma patients. Br J Cancer 103: 954-960, 2010.

27. Glazer ES, Piccirillo M, Albino V, Di Giacomo R, Palaia R, Mastro AA, Beneduce G, Castello G, De Rosa V, Petrillo A, et al: Phase II study of pegylated arginine deiminase for nonresectable and metastatic hepatocellular carcinoma. J Clin Oncol 28: 2220-2226, 2010.

28. Tomlinson BK, Thomson JA, Bomalaski JS, Diaz M, Akande T, Mahaffey N, Li T, Dutia MP, Kelly K, Gong IY, et al: Phase I trial of arginine deprivation therapy with ADI-PEG 20 plus docetaxel in patients with advanced malignant solid tumors. Clin Cancer Res 21: 2480-2486, 2015.

29. Hegedüs C, Truta-Feles K, Antalffy G, Várady G, Német K, Ozvegy-Laczka C, Kéri G, Orfi L, Szakács G, Settleman J, et al: Interaction of the EGFR inhibitors gefitinib, vandetanib, pelitinib and neratinib with the ABCG2 multidrug transporter: Implications for the emergence and reversal of cancer drug resistance. Biochem Pharmacol 84: 260-267, 2012.

30. Holohan C, Van Schaeybroeck S, Longley DB and Johnston PG: Cancer drug resistance: An evolving paradigm. Nat Rev Cancer 13: 714-726, 2013.

31. Ekins S, Bugrim A, Brovold L, Kirillov E, Nikolsky Y, Rakhmatulin E, Sorokina S, Ryabov A, Serebryiskaya T, Melnikov A, et al: Algorithms for network analysis in systemsADME/Tox using the MetaCore and MetaDrug platforms. Xenobiotica 36: 877-901, 2006.

32. Lamb J: The Connectivity Map: A new tool for biomedical research. Nat Rev Cancer 7: 54-60, 2007.

33. Lamb J, Crawford ED, Peck D, Modell JW, Blat IC, Wrobel MJ, Lerner J, Brunet JP, Subramanian A, Ross KN, et al: The Connectivity Map: Using gene-expression signatures to connect small molecules, genes, and disease. Science 313: 1929-1935, 2006.

34. Linnebank $M$, Tschiedel E, Häberle J, Linnebank A, Willenbring H, Kleijer WJ and Koch HG: Argininosuccinate lyase (ASL) deficiency: Mutation analysis in 27 patients and a completed structure of the human ASL gene. Hum Genet 111: 350-359, 2002.

35. Arsic N, Bendris N, Peter M, Begon-Pescia C, Rebouissou C, Gadéa G, Bouquier N, Bibeau F, Lemmers B and Blanchard JM: A novel function for Cyclin A2: Control of cell invasion via RhoA signaling. J Cell Biol 196: 147-162, 2012.

36. Bendris N, Lemmers B, Blanchard J-M and Arsic N: Cyclin A2 mutagenesis analysis: A new insight into $\mathrm{CDK}$ activation and cellular localization requirements. PLoS One 6: e22879, 2011. 
37. Jackman M, Kubota Y, den Elzen N, Hagting A and Pines J: Cyclin A- and cyclin E-Cdk complexes shuttle between the nucleus and the cytoplasm. Mol Biol Cell 13: 1030-1045, 2002.

38. Fux L, Ilan N, Sanderson RD and Vlodavsky I: Heparanase: Busy at the cell surface. Trends Biochem Sci 34: 511-519, 2009.

39. Ilan N, Elkin M and Vlodavsky I: Regulation, function and clinical significance of heparanase in cancer metastasis and angiogenesis. Int J Biochem Cell Biol 38: 2018-2039, 2006.

40. Gustafsson Sheppard N, Jarl L, Mahadessian D, Strittmatter L, Schmidt A, Madhusudan N, Tegnér J, Lundberg EK, Asplund A, Jain M, et al: The folate-coupled enzyme MTHFD2 is a nuclear protein and promotes cell proliferation. Sci Rep 5: 15029, 2015.

41. Lee JH, Jang H, Lee SM, Lee JE, Choi J, Kim TW, Cho EJ and Youn HD: ATP-citrate lyase regulates cellular senescence via an AMPK- and p53-dependent pathway. FEBS J 282: 361-371, 2015.

42. Yang W, Xia Y, Ji H, Zheng Y, Liang J, Huang W, Gao X, Aldape $\mathrm{K}$ and $\mathrm{Lu} Z$ : Nuclear PKM2 regulates $\beta$-catenin transactivation upon EGFR activation. Nature 480: 118-122, 2011.
43. Li B, Qiu B, Lee DS, Walton ZE, Ochocki JD, Mathew LK, Mancuso A, Gade TP, Keith B, Nissim I, et al: Fructose-1,6bisphosphatase opposes renal carcinoma progression. Nature 513: 251-255, 2014.

44. Jeffery CJ: Why study moonlighting proteins? Front Genet 6 : 211,2015

45. Cole SW and Sood AK: Molecular pathways: Beta-adrenergic signaling in cancer. Clin Cancer Res 18: 1201-1206, 2012.

46. Harris RE, Alshafie GA, Abou-Issa H and Seibert K: Chemoprevention of breast cancer in rats by celecoxib, a cyclooxygenase 2 inhibitor. Cancer Res 60: 2101-2103, 2000.

47. Jendrossek V: Targeting apoptosis pathways by Celecoxib in cancer. Cancer Lett 332: 313-324, 2013.

48. Zhao C-M, Hayakawa Y, Kodama Y, Muthupalani S, Westphalen CB, Andersen GT, Flatberg A, Johannessen H, Friedman RA, Renz BW, et al: Denervation suppresses gastric tumorigenesis. Sci Transl Med 6: 250ra115, 2014. 